\title{
Integrodifferential Inequalities Arising in the Theory of Differential Equations
}

\author{
Zareen A. Khan \\ Department of Mathematics, Princess Nora Bint Abdul Rahman University, Riyadh, Saudi Arabia \\ Correspondence should be addressed to Zareen A. Khan; dr.zareenkhan@ymail.com \\ Received 16 June 2016; Accepted 18 October 2016 \\ Academic Editor: Hatem Mejjaoli
}

Copyright (C) 2016 Zareen A. Khan. This is an open access article distributed under the Creative Commons Attribution License, which permits unrestricted use, distribution, and reproduction in any medium, provided the original work is properly cited.

The goal of this paper is to achieve some new results related to integrodifferential inequalities of one independent variable which can be applied as a study of qualitative and quantitative properties of solutions of some nonlinear integral equations.

\section{Introduction}

Integral and integrodifferential inequalities play a significant role in recent years by many authors [1-11], which provide an explicit bounds on the solutions of a class of differential and integral equations.

Lemma 1. Pachpatte (1995) studied the following useful integral inequality: Let $u(t), f(t)$, and $g(t)$ be nonnegative continuous functions defined on $R_{+}$and $c_{1}$ and $c_{2}$ be positive constants. If

$$
u(t) \leq\left(c_{1}+\int_{0}^{t} f(s) u(s) d s\right)\left(c_{2}+\int_{0}^{t} g(s) u(s) d s\right)
$$

for $t \in R_{+}$, where

$$
\begin{aligned}
& H(t)=1-c_{1} c_{2} \int_{0}^{t} R(s) Q(s) d s>0, \\
& R(t)=g(t) \int_{0}^{t} f(\sigma) d \sigma+f(t) \int_{0}^{t} g(\sigma) d \sigma, \\
& Q(t)=\exp \left(\int_{0}^{t}\left[c_{1} g(\sigma)+c_{2} f(\sigma)\right] d \sigma\right),
\end{aligned}
$$

for $t \in R_{+}$, then

$$
u(t) \leq\left[\frac{1}{H(t)}\right] c_{1} c_{2} Q(t),
$$

for all $t \in R_{+}$.

\section{Main Results}

Here by using Lemma 1, we establish some new results in the form of integrodifferential inequalities instead of integral inequality.

Theorem 2. Let $x(t), x^{*}(t), f(t)$, and $g(t)$ be nonnegative real valued continuous functions defined for $R_{+}=[0, \infty)$. Let $c_{1}$ and $c_{2}$ be positive constants. If

$$
\begin{aligned}
& x^{\cdot 2}(t) \\
& \leq\left(c_{1}+\int_{0}^{t} f(s) x^{\cdot 2}(s) d s\right)\left(c_{2}+\int_{0}^{t} g(s) x^{\cdot 2}(s) d s\right),
\end{aligned}
$$

for all $t \in R_{+}$, then

$$
x^{*}(t) \leq\left[\frac{1}{\sqrt{H(t)}}\right] \sqrt{\left(c_{1} c_{2}\right)} \sqrt{Q(t)},
$$

where $c_{1} c_{2} \geq 1$.

$H(t), R(t)$, and $Q(t)$ are defined as in (2), (3), and (4), respectively, for all $t \in R_{+}$.

Proof. Define a function $z^{2}(t)$ by the right-hand side of (6), such that

$$
\begin{aligned}
& z^{2}(t) \\
& =\left(c_{1}+\int_{0}^{t} f(s) x^{\cdot 2}(s) d s\right)\left(c_{2}+\int_{0}^{t} g(s) x^{\cdot 2}(s) d s\right),
\end{aligned}
$$


where

$$
z^{2}(0)=c_{1} c_{2}
$$

From (6) and (8), we get

$$
x^{\cdot 2}(t) \leq z^{2}(t) .
$$

By differentiating (8) and using the fact that

$$
x^{\cdot}(t) \leq z(t)
$$

we observe

$$
\begin{aligned}
2 z(t) & z^{\prime}(t) \\
\leq & \left(c_{1} g(t)+c_{2} f(t)\right) z^{2}(t) \\
& +\left(f(t) \int_{0}^{t} g(\sigma) d \sigma+g(t) \int_{0}^{t} f(\sigma) d \sigma\right) z^{4}(t)
\end{aligned}
$$

or

$$
\begin{aligned}
& 2 z^{-3}(t) z^{\prime}(t)-\left(c_{1} g(t)+c_{2} f(t)\right) z^{-2}(t) \\
& \quad \leq\left(f(t) \int_{0}^{t} g(\sigma) d \sigma+g(t) \int_{0}^{t} f(\sigma) d \sigma\right) .
\end{aligned}
$$

Let

$$
v(t)=z^{-2}(t) .
$$

Differentiating (14) with respect to $x$, we get

$$
v^{\prime}(t)=-2 z^{-3}(t) z^{\prime}(t),
$$

where

$$
v(0)=\left(c_{1} c_{2}\right)^{-1} .
$$

By substituting (14) and (15) in (13), we have

$$
v^{\prime}(t)+\left(c_{1} g(t)+c_{2} f(t)\right) v(t) \geq-R(t) .
$$

Inequality (17) implies the estimation for $v(t)$ and by using (16), we observe that

$$
\begin{aligned}
& v(t) \\
& \quad \geq\left(c_{1} c_{2}\right)^{-1} Q^{-1}(t)\left(1-\left(c_{1} c_{2}\right) \int_{0}^{t} R(s) Q(s) d s\right) d s,
\end{aligned}
$$

where $R(t)$ and $Q(t)$ are defined as in (3) and (4) and by applying (11) and (14) it is noticed that

$$
\dot{x}^{\cdot}(t) \leq\left[\frac{1}{\sqrt{H(t)}}\right] \sqrt{\left(c_{1} c_{2}\right)} \sqrt{Q(t)},
$$

where $H(t)$ is defined as in (2). This completes the proof.
Theorem 3. Let $x(t), x^{*}(t), f(t), g(t), c_{1}$, and $c_{2}$ be defined as in Theorem 2 for $R_{+}=[0, \infty)$. If

$$
\begin{aligned}
& x^{\cdot 2}(t) \\
& \leq\left(c_{1}+\int_{0}^{t} f(s) x^{\cdot}(s) d s\right)\left(c_{2}+\int_{0}^{t} g(s) x^{\cdot 2}(s) d s\right),
\end{aligned}
$$

for all $t \in R_{+}$, then

$$
\dot{x}^{\cdot}(t) \leq\left[\frac{1}{H(t)}\right] \sqrt{\left(c_{1} c_{2}\right)} Q(t),
$$

where $c_{1} c_{2} \geq 1$ and

$$
\begin{aligned}
& H(t)=1-\frac{\sqrt{\left(c_{1} c_{2}\right)}}{2} \int_{0}^{t} R(s) Q(s) d s>0, \\
& Q(t)=\exp \left(\left(\frac{1}{2}\right) \int_{0}^{t}\left[c_{1} g(\sigma)+c_{2} f(\sigma)\right] d \sigma\right)
\end{aligned}
$$

for all $t \in R_{+}$.

Proof. Define a function $z^{2}(t)$ by the right-hand side of (20), such that

$$
z^{2}(t)
$$

$$
=\left(c_{1}+\int_{0}^{t} f(s) x^{\cdot}(s) d s\right)\left(c_{2}+\int_{0}^{t} g(s) x^{\cdot 2}(s) d s\right),
$$

where

$$
z^{2}(0)=c_{1} c_{2}
$$

From (20) and (24), we get

$$
x^{\cdot 2}(t) \leq z^{2}(t) .
$$

By differentiating (24) and since $z(t)$ is monotone nondecreasing function for $t \in R_{+}$and using the fact that

$$
x^{\cdot}(t) \leq z(t)
$$

we observe that

$$
\begin{aligned}
& 2 z(t) z^{\prime}(t) \\
& \leq\left(c_{1} g(t)+c_{2} f(t)\right) z^{2}(t) \\
& \quad+\left(f(t) \int_{0}^{t} g(\sigma) d \sigma+g(t) \int_{0}^{t} f(\sigma) d \sigma\right) z^{3}(t)
\end{aligned}
$$

or

$$
\begin{aligned}
& 2 z^{-2}(t) z^{\prime}(t)-\left(c_{1} g(t)+c_{2} f(t)\right) z^{-1}(t) \\
& \quad \leq\left(f(t) \int_{0}^{t} g(\sigma) d \sigma+g(t) \int_{0}^{t} f(\sigma) d \sigma\right) .
\end{aligned}
$$


Let

$$
\begin{aligned}
& v(t)=z^{-1}(t) ; \\
& v(0)=\left(c_{1} c_{2}\right)^{-1 / 2} .
\end{aligned}
$$

By repeating the same steps from (14)-(18) in (29) with suitable modifications, the estimation for $v(t)$ implies

$$
\begin{aligned}
v(t) \geq & \left(c_{1} c_{2}\right)^{-1 / 2} Q^{-1}(t) \\
& \cdot\left(1-\frac{\sqrt{\left(c_{1} c_{2}\right)}}{2} \int_{0}^{t} R(s) Q(s) d s\right) d s .
\end{aligned}
$$

From (27) and (30) in (31), we get

$$
\dot{x}^{\cdot}(t) \leq\left[\frac{1}{H(t)}\right] \sqrt{\left(c_{1} c_{2}\right) Q(t)}
$$

for all $t \in R_{+}$, where $R(t), H(t)$, and $Q(t)$ are defined as in (3), (22), and (23), respectively. This completes the proof.

Theorem 4. Let $x(t), x^{*}(t), f(t), g(t), c_{1}$, and $c_{2}$ be defined as in Theorem 2 for $R_{+}=[0, \infty)$. If

$$
\begin{aligned}
& x^{\cdot p}(t) \\
& \leq\left(c_{1}+\int_{0}^{t} f(s) x^{\cdot p}(s) d s\right)\left(c_{2}+\int_{0}^{t} g(s) x^{\cdot}(s) d s\right),
\end{aligned}
$$

for all $t \in R_{+}$, then

$$
\dot{x}^{\cdot}(t) \leq\left[\frac{1}{H(t)}\right]\left(c_{1} c_{2}\right)^{1 / p} Q(t),
$$

where $c_{1} c_{2} \geq 1$ and $p>0$.

$$
\begin{aligned}
& H(t)=1-\frac{\left(c_{1} c_{2}\right)^{1 / p}}{p} \int_{0}^{t} R(s) Q(s) d s>0 \\
& Q(t)=\exp \left(\left(\frac{1}{p}\right) \int_{0}^{t}\left[c_{1} g(\sigma)+c_{2} f(\sigma)\right] d \sigma\right)
\end{aligned}
$$

for all $t \in R_{+}$.

Proof. Define a function $z^{p}(t)$ by the right-hand side of (33), such that

$$
\begin{aligned}
& z^{p}(t) \\
& =\left(c_{1}+\int_{0}^{t} f(s) x^{\cdot p}(s) d s\right)\left(c_{2}+\int_{0}^{t} g(s) x^{\cdot}(s) d s\right),
\end{aligned}
$$

where

$$
z^{p}(0)=c_{1} c_{2} .
$$

From (33) and (37), we get

$$
x^{\cdot p}(t) \leq z^{p}(t)
$$

or

$$
\dot{x}^{\cdot}(t) \leq z(t) .
$$

By differentiating (37) and since $z(t)$ is monotone nondecreasing function for $t \in R_{+}$, we observe that

$$
\begin{aligned}
& p z^{p-1}(t) z^{\prime}(t) \\
& \leq\left(c_{1} g(t)+c_{2} f(t)\right) z^{p}(t) \\
& \quad+\left(f(t) \int_{0}^{t} g(\sigma) d \sigma+g(t) \int_{0}^{t} f(\sigma) d \sigma\right) z^{p+1}(t)
\end{aligned}
$$

or

$$
\begin{aligned}
& p z^{-2}(t) z^{\prime}(t)-\left(c_{1} g(t)+c_{2} f(t)\right) z^{-1}(t) \\
& \quad \leq\left(f(t) \int_{0}^{t} g(\sigma) d \sigma+g(t) \int_{0}^{t} f(\sigma) d \sigma\right) .
\end{aligned}
$$

Let

$$
\begin{aligned}
& v(t)=z^{-1}(t) ; \\
& v(0)=\left(c_{1} c_{2}\right)^{-1 / p} .
\end{aligned}
$$

By repeating the same steps from (14)-(18) in (42) with suitable modifications, the estimation for $v(t)$ implies

$$
\begin{aligned}
v(t) \geq & \left(c_{1} c_{2}\right)^{-1 / p} Q^{-1}(t) \\
& \cdot\left(1-\frac{\left(c_{1} c_{2}\right)^{1 / p}}{p} \int_{0}^{t} R(s) Q(s) d s\right) d s .
\end{aligned}
$$

From (40) and (43) in (44), we get

$$
x^{\bullet}(t) \leq\left[\frac{1}{H(t)}\right]\left(c_{1} c_{2}\right)^{1 / p} Q(t),
$$

for all $t \in R_{+}$, where $R(t), H(t)$, and $Q(t)$ are defined as in (3), (35), and (36), respectively. This completes the proof.

Theorem 5. Let $x(t), x^{*}(t), f(t), g(t), c_{1}$, and $c_{2}$ be defined as in Theorem 2 for $R_{+}=[0, \infty)$. If

$$
\begin{aligned}
x^{\cdot p}(t) \leq & \left(c_{1}+\int_{0}^{t} f(s)\left[x^{\cdot}(t)+x^{\cdot p}(s)\right] d s\right) \\
& \cdot\left(c_{2}+\int_{0}^{t} g(s) x^{\cdot}(s) d s\right),
\end{aligned}
$$

for all $t \in R_{+}$, then

$$
x^{\cdot}(t) \leq\left[\frac{1}{H(t)}\right]\left(c_{1} c_{2}\right)^{1 / p} Q(t),
$$

where $c_{1} c_{2} \geq 1$ and $p>0$. 


$$
\begin{aligned}
& H(t)=1-\frac{\left(c_{1} c_{2}\right)^{1 / p}}{p} \int_{0}^{t} R(s) Q(s) d s>0 \\
& Q(t)=\exp \left(\left(\frac{1}{p}\right) \int_{0}^{t}\left[c_{1} g(\sigma)+2 c_{2} f(\sigma)+f(\sigma) \int_{0}^{s} g(\eta) d \eta+g(\sigma) \int_{0}^{s} f(\eta) d \eta\right] d \sigma\right)
\end{aligned}
$$

for all $t \in R_{+}$.

Proof. Define a function $z^{P}(t)$ by the right-hand side of (46), such that

$$
\begin{aligned}
z^{p}(t)= & \left(c_{1}+\int_{0}^{t} f(s)\left[x^{\bullet}(t)+x^{\cdot p}(s)\right] d s\right) \\
& \cdot\left(c_{2}+\int_{0}^{t} g(s) x^{\bullet}(s) d s\right),
\end{aligned}
$$

where

$$
z^{p}(0)=c_{1} c_{2}
$$

From (46) and (50), we get

$$
x^{\cdot p}(t) \leq z^{p}(t)
$$

or

$$
x^{\bullet}(t) \leq z(t) .
$$

By differentiating (50) and since $z(t)$ is monotone nondecreasing function for $t \in R_{+}$, we observe that

$$
\begin{aligned}
& p z^{p-1}(t) z^{\prime}(t) \leq\left(c_{1} g(\sigma)+2 c_{2} f(\sigma)\right. \\
& \left.\quad+f(\sigma) \int_{0}^{s} g(\eta) d \eta+g(\sigma) \int_{0}^{s} f(\eta) d \eta\right) z^{p}(t) \\
& \quad+R(t) z^{p+1}(t)
\end{aligned}
$$

or

$$
\begin{aligned}
& p z^{-2}(t) z^{\prime}(t)-\left(c_{1} g(\sigma)+2 c_{2} f(\sigma)\right. \\
& \left.\quad+f(\sigma) \int_{0}^{s} g(\eta) d \eta+g(\sigma) \int_{0}^{s} f(\eta) d \eta\right) z^{-1}(t) \\
& \quad \leq R(t) .
\end{aligned}
$$

Let

$$
\begin{aligned}
& v(t)=z^{-1}(t) ; \\
& v(0)=\left(c_{1} c_{2}\right)^{-1 / p} .
\end{aligned}
$$

By repeating the same steps from (14)-(18) in (55) with suitable modifications, the estimation for $v(t)$ implies

$$
\begin{aligned}
v(t) \geq & \left(c_{1} c_{2}\right)^{-1 / p} Q^{-1}(t) \\
& \cdot\left(1-\frac{\left(c_{1} c_{2}\right)^{1 / p}}{p} \int_{0}^{t} R(s) Q(s) d s\right) d s .
\end{aligned}
$$

From (53) and (56) in (57), we get

$$
x^{\cdot}(t) \leq\left[\frac{1}{H(t)}\right]\left(c_{1} c_{2}\right)^{1 / p} Q(t),
$$

for all $t \in R_{+}$, where $R(t), H(t)$, and $Q(t)$ are defined as in (3), (48), and (49), respectively. This completes the proof.

Theorem 6. Let $x(t), x^{\bullet}(t), f(t), g(t), c_{1}$, and $c_{2}$ be defined as in Theorem 2 for $R_{+}=[0, \infty)$. If

$$
\begin{aligned}
& x^{\bullet p}(t) \\
& \leq\left(c_{1}+\int_{0}^{t} f(s) x^{\bullet q}(s) d s\right)\left(c_{2}+\int_{0}^{t} g(s) x^{\bullet}(s) d s\right),
\end{aligned}
$$

for all $t \in R_{+}$, then

$$
\begin{aligned}
x^{\bullet}(t) & \leq\left[\frac{1}{Q(t)}\right]^{1 /(p-q)}\left[\left(c_{1} c_{2}\right)^{p-q / p}\right. \\
+ & \left.\left(\frac{p-q}{p}\right) \int_{0}^{t}\left(c_{1} f(s)+c_{2} g(s)\right) Q(s) d s\right]^{1 /(p-q)},
\end{aligned}
$$

where $c_{1} c_{2} \geq 1, p>q \geq 1$, and $p-q \geq 1$.

$$
Q(t)=\exp \left(\frac{-(p-q)}{p} \int_{0}^{t} R(s) d s\right)
$$

for all $t \in R_{+}$.

Proof. Define a function $z^{P}(t)$ by the right-hand side of (59), such that

$$
\begin{aligned}
& z^{p}(t) \\
& =\left(c_{1}+\int_{0}^{t} f(s) x^{\bullet q}(s) d s\right)\left(c_{2}+\int_{0}^{t} g(s) x^{\bullet}(s) d s\right),
\end{aligned}
$$

where

$$
z^{p}(0)=c_{1} c_{2}
$$

From (59) and (62), we get

$$
x^{\bullet p}(t) \leq z^{p}(t)
$$

or

$$
x^{\bullet}(t) \leq z(t) .
$$


By differentiating (62) and since $z(t)$ is monotone nondecreasing function for $t \in R_{+}$, we observe that

$$
\begin{aligned}
p z^{p-1}(t) z^{\prime}(t) & \\
\leq & \left(c_{1} g(t)+c_{2} f(t)\right) z^{q}(t) \\
& +\left(f(t) \int_{0}^{t} g(\sigma) d \sigma+g(t) \int_{0}^{t} f(\sigma) d \sigma\right) z^{1+q}(t)
\end{aligned}
$$

or

$$
p z^{p-q-1}(t) z^{\prime}(t)-R(t) z^{p-q}(t) \leq\left(c_{1} g(t)+c_{2} f(t)\right) .
$$

Let

$$
\begin{aligned}
& v(t)=z^{p-q}(t) ; \\
& v(0)=\left(c_{1} c_{2}\right)^{(p-q) / p} .
\end{aligned}
$$

By repeating the same steps from (14)-(18) in (67) with suitable modifications, the estimation for $v(t)$ implies

$$
\begin{aligned}
& v(t) \leq\left[\frac{1}{Q(t)}\right]^{1 /(p-q)}\left[\left(c_{1} c_{2}\right)^{p-q / p}\right. \\
&\left.+\left(\frac{p-q}{p}\right) \int_{0}^{t}\left(c_{1} f(s)+c_{2} g(s)\right) Q(s) d s\right]^{1 /(p-q)} .
\end{aligned}
$$

From (65) and (68) in (69), we get

$$
\begin{aligned}
x^{\cdot}(t) & \leq\left[\frac{1}{Q(t)}\right]^{1 /(p-q)}\left[\left(c_{1} c_{2}\right)^{p-q / p}\right. \\
& \left.+\left(\frac{p-q}{p}\right) \int_{0}^{t}\left(c_{1} f(s)+c_{2} g(s)\right) Q(s) d s\right]^{1 /(p-q)},
\end{aligned}
$$

for all $t \in R_{+}$, where $R(t)$ and $Q(t)$ are defined as in (3) and (61), respectively. This completes the proof.

\section{Application}

As an application, the explicit bounds of some of the integral inequalities can be found by some examples.

Example 1. Let us consider the explicit bound on the solution of the nonlinear integrodifferential equation

$$
\begin{aligned}
x^{\cdot 2} & (t) \\
& \leq\left(1+\int_{0}^{t} f(s) x^{\cdot 2}(s) d s\right)\left(1+\int_{0}^{t} g(s) x^{\bullet}(s) d s\right),
\end{aligned}
$$

where $x^{\bullet}(s)$ is a nonnegative real valued continuous function and every solution of $x^{\bullet}(s)$ of (71) exists for $R_{+}$. that

By using the application of Theorem 4 to (71), we observe

$$
x^{\cdot}(t) \leq\left[\frac{1}{H(t)}\right] Q(t)
$$

where

$$
\begin{aligned}
R(t) & =\int_{0}^{t} d s+\int_{0}^{t} d s=2 t \\
H(t) & =1-\frac{1}{2} \int_{0}^{t} R(s) Q(s) d s>0=1-\frac{1}{2} \int_{0}^{t} 2 s e^{s} d s \\
& =1-\int_{0}^{t} s e^{s} d s=-t e^{t}+e^{t}, \\
Q(t) & =\exp \left(\left(\frac{1}{2}\right) \int_{0}^{t} 2 d s\right)=\exp \left(\int_{0}^{t} d s\right)=e^{t} .
\end{aligned}
$$

Therefore the right-hand side of (74) provides the bound of the solution of (75) of known quantities

$$
x^{\cdot} t \leq \frac{e^{t}}{e^{t}+t e^{t}} \leq \frac{1}{1-t}
$$

for $0 \leq t<1$.

Example 2. Let us consider the nonlinear integrodifferential equation of the form

$$
\begin{aligned}
x^{\cdot p}(t) \leq & \left(1+\int_{0}^{t} f(s)\left[x^{\bullet}(t)+x^{\cdot 2}(s)\right] d s\right) \\
& \cdot\left(1+\int_{0}^{t} g(s) x^{\cdot}(s) d s\right),
\end{aligned}
$$

where $x^{\bullet}(s)$ is a nonnegative real valued continuous function and every solution of $x^{\bullet}(s)$ of (77) exists for $R_{+}$.

By using the application of Theorem 5 to (77), we observe that

$$
x^{\bullet}(t) \leq\left[\frac{1}{H(t)}\right] Q(t),
$$

where

$$
\begin{aligned}
R(t) & =\int_{0}^{t} d s+\int_{0}^{t} d s=2 t \\
H(t) & =1-\frac{1}{2} \int_{0}^{t} R(s) Q(s) d s>0 \\
& =1-\frac{1}{2} \int_{0}^{t} 2 s e^{(3 / 2) s+(1 / 2) s^{2}} d s \\
& =1-\int_{0}^{t} s e^{\left((3 / 2) s+(1 / 2) s^{2}\right)} d s, \\
Q(t) & =\exp \left(\left(\frac{1}{2}\right) \int_{0}^{t}\left(1+2+\int_{0}^{t} d \sigma+\int_{0}^{t} d \sigma\right) d s\right) \\
& =\exp \frac{1}{2}\left(3 t+t^{2}\right)=e^{\left((3 / 2) t+(1 / 2) t^{2}\right)} .
\end{aligned}
$$

Therefore the right-hand side of (80) provides the bound of the solution of (77) of known quantities

$$
x^{\bullet}(t) \leq \frac{e^{\left((3 / 2) t+(1 / 2) t^{2}\right)}}{1-\int_{0}^{t} s e^{\left((3 / 2) s+(1 / 2) s^{2}\right)} d s}
$$

for $0 \leq t<1$. 
Example 3. Now let us consider the boundedness and asymptotic behaviour of the solutions of nonlinear Volterra integrodifferential inequality of the form

$$
\begin{aligned}
x^{\cdot p}(t)= & \left(a_{1}(t)+\int_{0}^{t} A(t-s) x^{\bullet q}(s) d s\right) \\
& \cdot\left(a_{2}(t)+\int_{0}^{t} B(t-s) x^{\bullet}(s) d s\right) ;
\end{aligned}
$$

$x^{\bullet}(t)$ is nonnegative real valued continuous function defined on $R_{+}$and $a_{1}, a_{2}, A, B$ are real valued continuous function defined on $R_{+}$.

We assume that every solution of $x^{\circ}(t)$ in (81) exists on $R_{+}$, and $p$ and $q$ are defined as in Theorem 6. Define the following hypotheses on the function of (81) as

$$
\begin{aligned}
& \left|a_{1}(t)\right| \leq c_{1}, \\
& \left|a_{2}(t)\right| \leq c_{2} .
\end{aligned}
$$

Also

$$
\begin{aligned}
& |A(t-s)| \leq M_{1} f_{1}(s) \\
& |B(t-s)| \leq N_{1} g_{1}(s) \\
& D(t)=\left[\frac{1}{Q(t)}\right]^{1 /(p-q)}\left[\left(c_{1} c_{2}\right)^{p-q / p}\right. \\
& \left.\quad+\left(\frac{p-q}{p}\right) \int_{0}^{t}\left(c_{1} g(s)+c_{2} f(s)\right) Q(s) d s\right]^{1 /(p-q)} \\
& \quad<\infty
\end{aligned}
$$

for all $0 \leq s \leq t, s, t \in R_{+} . M, N, c_{1}, c_{2}$ are nonnegative real constants and $f_{1}, g_{1}$ are nonnegative real valued continuous function defined on $R_{+}$.

Proof. For the boundedness of the solution of nonlinear integrodifferential equation (83), let us suppose that the hypotheses (84), (85), and (86) are satisfied and let $x^{\circ}(t)$ be a solution of (83); then we observe that

$$
\begin{aligned}
\left|x^{\cdot p}(t)\right| \leq & \left(\left|a_{1}(t)\right|+\int_{0}^{t}|A(t-s)|\left|x^{\cdot q}(s)\right| d s\right) \\
& \cdot\left(\left|a_{2}(t)\right|+\int_{0}^{t}|B(t-s)|\left|x^{\bullet}(s)\right| d s\right) .
\end{aligned}
$$

Replacing $f$ by $M_{1} f_{1}$ and $g$ by $N_{1} g_{1}$ and applying the same proof with some modifications of Theorem 6 in (88) and with $R(t)$ and $Q(t)$ being the same as defined in Theorem 6, we noticed that every solution of $x^{\bullet}(t)$ of $(88)$ that exists on $R_{+}$is bounded; that is,

$$
\left|x^{\cdot p}(t)\right| \leq D(t) \text {. }
$$

For the asymptotic behaviour of the solution of nonlinear integrodifferential equation (83), assume the following hypotheses

$$
\begin{aligned}
& \left|a_{1}(t)\right| \leq c_{1} e^{-\mu t}, \\
& \left|a_{2}(t)\right| \leq c_{2} e^{-\mu t} .
\end{aligned}
$$

Also

$$
\begin{gathered}
|A(t-s)| \leq M_{1} f_{1}(s) e^{-\mu(t-2 s)}, \\
|B(t-s)| \leq N_{1} g_{1}(s) e^{-\mu(t-2 s)}
\end{gathered}
$$

are satisfied. Let $x^{\bullet}(t)$ be a solution of (83); then

$$
\begin{aligned}
\left|x^{\cdot p}(t)\right| \leq & e^{-2 \mu t}\left(c_{1}+\int_{0}^{t} M_{1} f_{1}(s)\left|x^{\cdot q}(s)\right| e^{2 \mu s} d s\right) \\
& \cdot\left(c_{2}+\int_{0}^{t} N_{1} g_{1}(s)\left|x^{\cdot}(s)\right| e^{2 \mu s} d s\right) .
\end{aligned}
$$

Let $z^{m}(t)$ be $\left|x^{\bullet p}(t)\right| e^{2 \mu t}$ for $m>0$ in (92) and by applying the same proof with some changes of Theorem 6 in (92), we get

$$
z(t) \leq D(t) \Longrightarrow\left|x^{\cdot}(t)\right| \leq D(t) e^{-2 \mu t}
$$

Therefore the solution $x^{\bullet}(t)$ of (83) is asymptotically stable.

\section{Competing Interests}

There is no conflict of interests regarding the publication of this paper.

\section{References}

[1] D. Bainov and P. Simeonov, Integral Inequalities and Applications, vol. 57 of Mathematics and Its Applications, Kluwer Academic Publishers Group, Dordrecht, The Netherlands, 1992.

[2] R. Bellman, "The stability of solutions of linear differential equations," Duke Mathematical Journal, vol. 10, pp. 643-647, 1943.

[3] F. Jiang and F. Meng, "Explicit bounds on some new nonlinear integral inequalities with delay," Journal of Computational and Applied Mathematics, vol. 205, no. 1, pp. 479-486, 2007.

[4] H. T. Gronwall, "Note on the derivatives with respect to a parameter of the solutions of a system of differential equations," The Annals of Mathematics, vol. 2, pp. 292-296, 1918.

[5] O. Lipovan, "Integral inequalities for retarded Volterra equations," Journal of Mathematical Analysis and Applications, vol. 322, no. 1, pp. 349-358, 2006.

[6] B. G. Pachpatte, "On some fundamental integral inequalities and their discrete analogues," Journal of Inequalities in Pure \& Applied Mathematics, vol. 2, no. 2, pp. 1-13, 2001.

[7] B. G. Pachpatte, "On a new inequality suggested by the study of certain epidemic models," Journal of Mathematical Analysis and Applications, vol. 195, no. 3, pp. 638-644, 1995.

[8] B. G. Pachpatte, "A note on Gronwall-Bellman inequality," Journal of Mathematical Analysis and Applications, vol. 44, pp. 758-762, 1973.

[9] B. G. Pachpatte, "Integral inequalities of Gronwall-Bellman type and their applications," Journal of Mathematical and Physical Sciences, vol. 8, pp. 309-318, 1974.

[10] B. G. Pachpatte, Inequalities for Differential and Integral Equations, Academic Press INC, 1998.

[11] Y.-H. Kim, "Gronwall, Bellman and Pachpatte type integral inequalities with applications," Nonlinear Analysis: Theory, Methods \& Applications, vol. 71, no. 12, pp. e2641-e2656, 2009. 


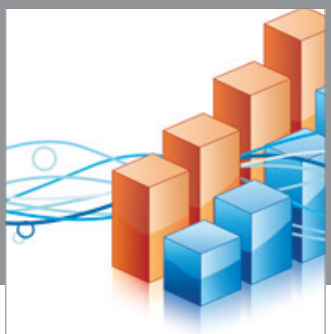

Advances in

Operations Research

vatem alat4

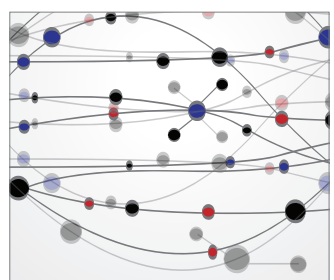

\section{The Scientific} World Journal
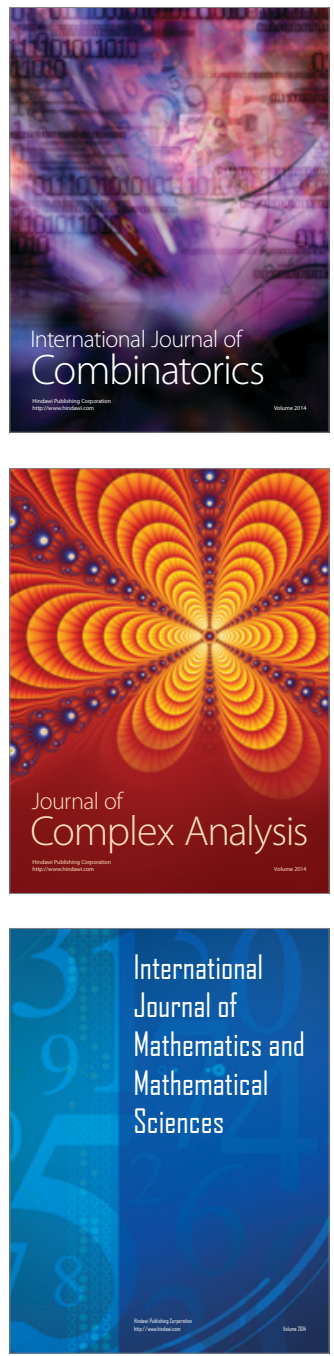
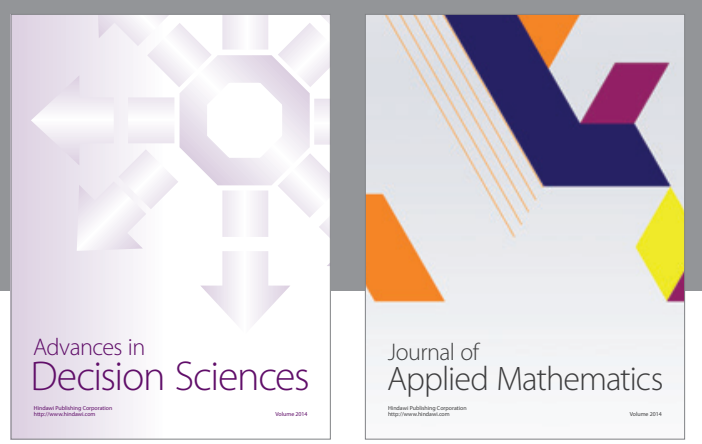

Algebra

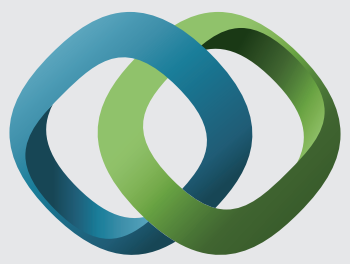

\section{Hindawi}

Submit your manuscripts at

http://www.hindawi.com
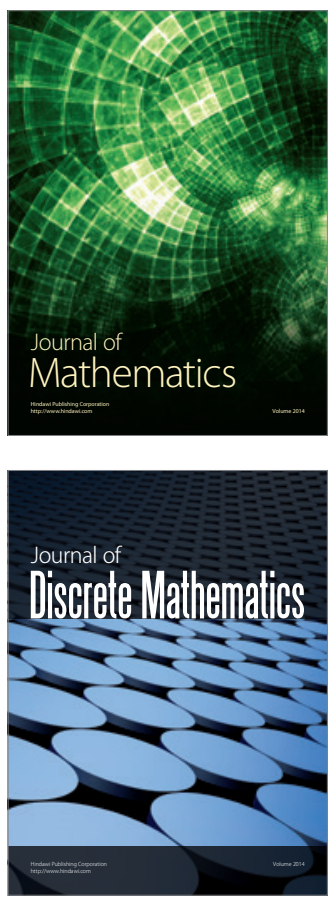

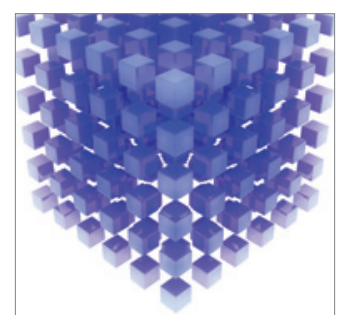

Mathematical Problems in Engineering
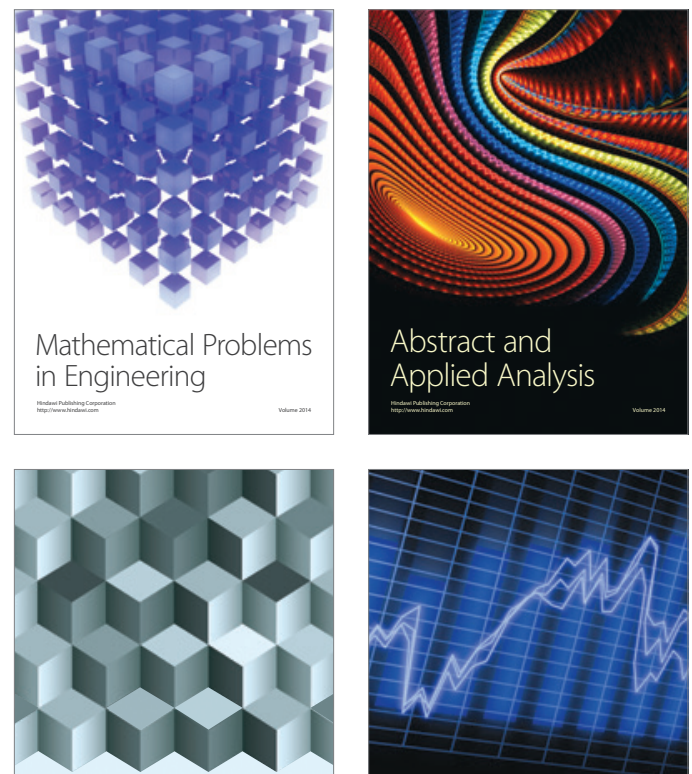

Journal of

Function Spaces

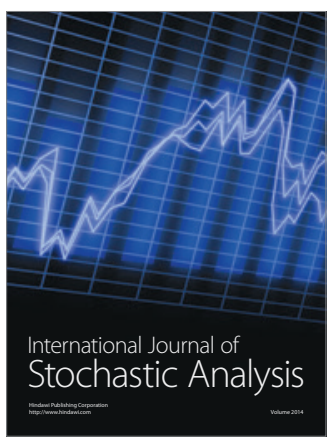

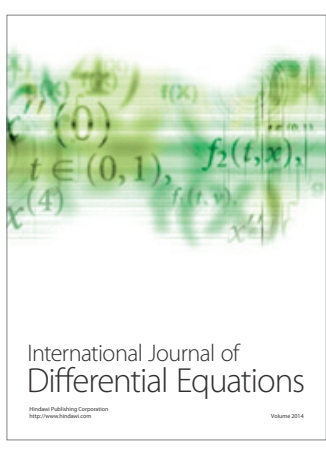
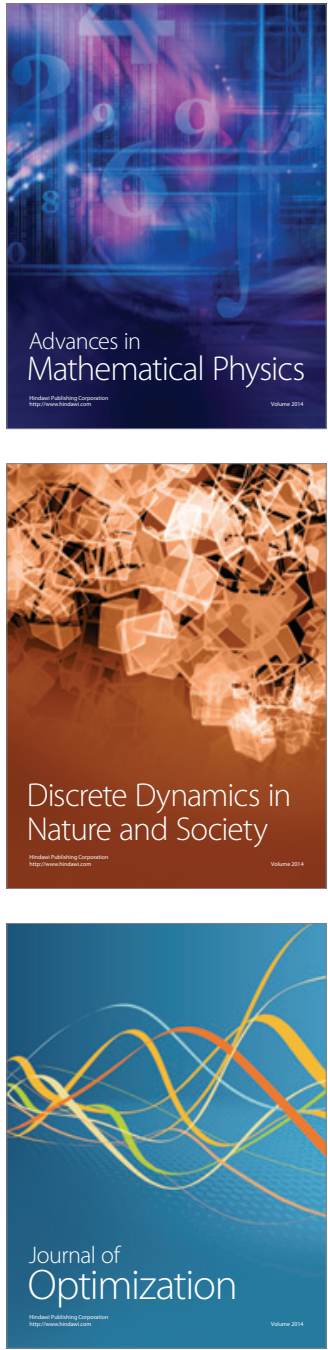